\title{
An efficient anonymous authentication protocol in multiple server communication networks (EAAM)
}

\author{
An Braeken • Pardeep Kumar • \\ Madhusanka Liyanage • Thi Kim Hue Ta
}

the date of receipt and acceptance should be inserted later

\begin{abstract}
In a multi-server authentication environment, a user only needs to register once at a central registration place before accessing the different services on the different registered servers. Both, from a user point of view as for the management and maintenance of the infrastructure, these type of environments become more and more popular. Smart card or smart phone based approaches lead to more secure systems because they offer two or threefactor authentication, based on the strict combination of the user's password, the user's biometrics and the possession of the device.

In this paper, we propose an efficient anonymous authentication protocol in multiple server communication networks, called the EAAM protocol, which is able to establish user anonymity, mutual authentication, and resistance against known security attacks. The novelty of the proposed scheme is that it does not require a secure channel during the registration between the user and the registration center and is resistant to a curious but honest registration system. These features are established in a highly efficient way with the minimum amount of communication flows between user and server during the establishment of the secret shared key and by using light-weight cryptographic techniques such as Chebyshev chaotic map techniques and symmetric
\end{abstract}

An Braeken

INDI and ETRO Department

Vrije Universiteit Brussel, Belgium

Pardeep Kumar

Department of Computer Science

University of Oxford

Madhusanka Liyanage

Centre for Wireless Communications

University of Oulu, Finland

Thi Kim Hue Ta

School of Electronics and Telecommunications

Hanoi University of Science and Technology, Viet Nam 
key cryptography. The performance and security of the protocol are analyzed and compared with the latest new proposals in this field.

Keywords Multi-server authentication · Anonymity · Chaotic maps

\section{Introduction}

Enterprises make more and more use of multi-server authentication environments, enabling their employees to get access with one single registration to different services running on different servers in the infrastructure. Also several e-government services can be currently accessed by the user through one single registration.

This is a significant improvement, compared with the classical authentication method where the user has to send to each of these services an identity and hashed password, which is then compared with the stored verification table at server side. Such method encompasses several security problems and requires huge storage capacities. In 2000, Hwang et al. [1] introduced the smart card based authentication. Here, a registration center (RC) activates the card with some secret information, which is (partly) shared with the registered servers. It can be seen as a two-factor authentication since both the possession of the smart card and the knowledge of the password is required. During the last decade, a lot of research has been performed on developing authentication protocols for this type of setting, adding more and more additional security features and becoming more and more practical. For instance, we can identify three main classes based on the used cryptographic mechanisms: (i) symmetric key based, (ii) public key based by means of elliptic curve cryptography (ECC) or chaotic maps, and (iii) bilinear pairing based systems. However, the main drawback in a symmetric key system is that it does not allow the construction of key material without the secure channel and it offers less resistance against insider attacks. The bilinear pairings systems are highly demanding operations (in terms of time complexity, e.g., point multiplications, etc. ), compared with the operations of the other classes, as shown in Table 3 of [2]. For the public key based systems, the usage of chaotic maps is about three times more efficient than the classical elliptic curve based operations [3]. Most of the literature provides mainly the classical security properties but requires a secure channel at the user registration, which is not practical always. Moreover, many of the schemes consist of more than three, often five different communication flows, and thus enormously increase the complexity. Another vulnerability in such schemes is that a registration center could perform the required operations, honestly, but it may reveal information for its own purposes. Therefore, these schemes cannot withstand against the honest-but-curious attack. To end this, we assumed that a security protocol must satisfy the following security features in the multi-server environment:

- Anonymity to the outsider: Identity is one of the sensitive personal information that could be leaked over the communication to track the user 
or device activities. Therefore, anonymity is highly required to provide the anonymous communication that can protect from such identity leaks.

- Anonymity or identity derivation of user by the server: This security property depends on the type of service is being offered. In some cases, it is beneficial that the identity of the user is not leaked to the server for the privacy protection of the user. The server only knows that the user is approved by the RC. However, the server should be ensured of the claimed identity of the user for the services such as payment activities.

- Unlinkability: The multi server communication network, two or more items of interest from an attacker's perspective means that the attacker can sufficiently link whether these items are related to a particular user or device over the communication. Therefore, it is paramount that different messages cannot be linked to the same user by any outsider collecting the transmitted messages.

- Mutual authentication: Both the user and the server could authenticate each other and contribute to the construction of the secret key.

- Multiple servers: For the sake of user-friendliness, a user can access multiple servers with the same credentials, obtained during the registration phase.

- Known key security: In the multi server communications, it is possible that an attacker or malevolent user could determine the session key for the ongoing communication. It is therefore, desirable that even if the secret session keys between the user and the server are revealed, no further information can be obtained from the private keys of user and the server.

- Perfect forward secrecy: Even if the longterm private keys of the user or the server are leaked, the previously derived secret session keys between the user and the server cannot be revealed.

- Resistance against known attacks: The scheme can withstand insider attack, stolen device attack, offline guessing attack, replay attack, user impersonation attack, and man-in-the-middle attack.

- Resistance against curious but honest RC: The RC is not able to derive the secret session key generated by the user and the server, and thus can not store the sensitive information of the user. Curious means that the $\mathrm{RC}$ will perform the required operations, but that it may reveal or misuse information for its own benefit.

- Avoidance of the secure channel: No secure channel is required between the user and the registration center during the registration phase.

Contribution: Considering the above security properties, we propose a new and efficient anonymous authentication protocol in the multiple server communication networks (EAAM). The proposed EAAM is based on the Chebyshev chaotic map operations and required only two communication phases for the construction of the secret session key, which is quite efficient. In order to authenticate the user, the EAAM uses a smart phone with installed biometric feature identification, instead of a smart card with a corresponding smart reader to perform the authentication. Moreover, the performance and 
security of the EAAM are analyzed and compared with the recently proposed literature in this field.

The rest of the paper is structured as follows. Section 2 presents related work. In Section 3, we give some background on the operations and the system setting. Section 4 describes our protocol. In Section 5 and 6 , we analyse the security and performance of the protocol respectively. Finally, we conclude the paper in Section 7.

\section{Related work}

Smart card based authentication mechanisms are proposed for both single server and multiple server environments [4]. They are either two-factor or three-factor based. However, as the biometrics related information is used in the same way as the password, the two-factor based schemes can be in most of the cases easily extended to three-factor based schemes. In [5], a generic framework to upgrade two-factor authentication schemes to three-factor ones using the concept of fuzzy extractor [6] is explained. There exists an enormous amount of papers on these type of protocols, e.g. a detailed survey can be found in [4]. We will limit the discussion on the latest generation of multiserver authentication protocols, which offer in addition user anonymity.

We can basically distinguish three types of classes in these authentication schemes: symmetric key based schemes, public key based schemes, and bilinear pairing based schemes, as follows.

Symmetric key based schemes - many symmetric key based schemes are also proposed in literature [15-24]. Almost all of them have been shown to be vulnerable to one or more of the following attacks: insider attacks, impersonation attacks, and stolen smart card attacks. Except for $[16,23,24]$, no attacks have been mentioned yet. In [23], the proposed protocol contains three communication rounds, and in [16] the protocol is missing the unlinkability feature. The system in [24] is currently the most efficient and complete system, given the possibilities that can be obtained with symmetric key operations. For instance, these systems cannot be resistant to an insider attack where one compromised user and server collaborate. They also require a secure channel during registration and the $\mathrm{RC}$ can always follow every communication between the user and the server, due to the inherent properties of symmetric key cryptography.

Public key based schemes- This is the second class where two main mechanisms are distinguished as the ECC and the Chebychev polynomials. For the ECC based systems, we distinguish in literature [25-33]. All these systems have been shown to be insecure $[25,26,28-30,32]$ or perform the authentication request through the $\mathrm{RC}$ and thus require five communication phases [31,33]. In [27] a secure and two-round scheme is presented, but although it is mentioned as a multi-server authentication protocol, unique credentials are required for each server, making it highly inefficient. For the systems using the Chebychev polynomials as trapdoor mechanism, several schemes have been proposed that 
without the usage of a RC $[34-39,13,14,40,41]$. However, many of them are vulnerable to different types of attacks (insider attacks, man-in-the-middle attacks, etc.) as shown in [34-39]. The protocols without reported attacks in this list are currently $[13,14,40,41]$. For the classical setting with RC and servers, consisting of only two communication rounds, we identify the references [4244]. The protocol in [42] is clearly not resistant against insider attacks as the $\mathrm{RC}$ and all the servers share the same secret key. The scheme in [43] allows to trace the user. Finally, as the RC derives in both $[43,44]$ the secret key material of the user and the servers, both protocols are not prone against a curious RC and do require a secure channel during the registration.

Biliner pairing based schemes - The references [7-10,13,14] belong to the third class. In [14], it has been shown that the proposed scheme was the most complete with respect to security features and computational efficiency, compared to the other bilinear protocols. Still, these type of schemes do not offer more security features as the others, all possess at least three communication phases and are very expensive due to the inherent computational demanding bilinear operation.

To conclude, as far as the authors are aware, there are currently no protocols available in literature, that satisfy besides the classical security features as anonymity, unlinkability, perfect forward secrecy, known key secrecy, that are in addition resistant to a curious and honest RC. As privacy of the user is becoming more and more important, it is an interesting feature to provide to the user. In addition, we also include the mechanisms that avoid the need for a secure channel, which is in practice difficult to realize. Moreover, as efficiency both in terms of latency, computational complexity and energy consumption is very relevant to mobile devices, it is important to limit the number of required communication rounds to a minimum and to use the most efficient security operations.

\section{Preliminaries}

\subsection{Chebyshev chaotic map}

The basics of the Chebyshev chaotic maps are now shortly discussed. For more details, we refer to $[45,46]$. The Chebyshev polynomials are defined as:

$$
T_{n}(x):[-1,1] \rightarrow[-1,1]: T_{n}(x)=\cos (n \arccos (x)), n \in \mathbb{N}
$$

To enhance the properties of Chebyshev chaotic maps, Zhang [47] proposed an improved Chebyshev polynomial defined on the domain $\mathbb{R}$, where the modulo of the result is taken over a large prime number $p$. The following recurrent relation can be used to determine these Chebyshev polynomial maps $T_{n}: R \rightarrow R$ with $n \geq 2$

$$
T_{n}(x)=2 x T_{n-1}(x)-T_{n-2}(x) \quad \bmod p, \text { given that } T_{0}(x)=1, T_{1}(x)=x .
$$


The improved Chebyshev polynomials satisfy the semi-group property, i.e., the equation $T_{v}\left(T_{u}(x)\right)=T_{u}\left(T_{v}(x)\right) \bmod p$ holds for $u, v \in \mathbb{N}$ and $x \in \mathbb{R}$. The interesting aspect at the Chebyshev polynomials is that they can be used in the discrete logarithm (DL) problem and the computational Diffie-Hellman $(\mathrm{CDH})$ problem.

DL problem: Given two elements $x$ and $y$, it is computationally infeasible to find an integer $r$ such that the equation $T_{r}(x)=y(\bmod p)$ holds.

CDH problem: Given $x, T_{r}(x), T_{s}(x)$, it is computationally infeasible to compute the result of the computations $t_{r s}=T_{r s}(x)=T_{r}\left(T_{s}(x)\right)=T_{s}\left(T_{r}(x)\right)=$ $T_{s r}(x)=t_{s r} \quad(\bmod p)$.

Also a signature scheme [48] based on the Chebyshev polynomials has been proposed.

\subsection{Usage of biometrics data}

When the biometric data of a user is captured, there are inherently different types of noise included. Consequently, repeated acquisitions of a biometric feature may lead to different, but small variations in this data. When using a classical hash function (eg. SHA1, SHA2) or encryption function (eg. AES) on these different variations, a completely different result will be obtained.

In order to overcome this problem, mechanisms such as fuzzy extractor [49], biohashing [50] or cancelable biometrics [51] are applied to the biometric data, allowing the derivation of a fixed user-specific random vector. We refer to the literature for more details on these mechanisms. Therefore, when we mention biometric data of a user in the protocol, we mean the output which is generated by one of the above mentioned mechanisms.

\subsection{System setting}

The main entities in the system are as follows.

- The user and its smart phone, which is provided with biometric feature extraction. The smart phone possesses a secure tamper resistant module (TRM) to store user specific, temporary key material, and no long term system security parameters.

- There are multiple servers, each offering a particular service.

- The central authority (CA) checks the identity of the users or servers, requesting to get access to the infrastructure. If positive, certificates are created for the user and server to register at the registration center. We assume a trustworthy CA as it is the basis of the whole system.

- The registration center $(\mathrm{RC})$ is responsible for the registration of the servers and users, based on a valid identification performed by the CA. We assume an honest, but curious RC.

We assume that the required cryptographic functions and parameters are implemented in each entity participating in the scheme. We further assume that 
Table 1: Abbreviations and notations

\begin{tabular}{ll}
\hline$U, U_{d}$ & User and User's device \\
TRM & Tamper resistant module in user's device \\
RC, S & Registration center and Server \\
CA & Central authority \\
$x$ & Public system parameter, the seed generating Chebyshev chaotic map \\
$y$ & Secret parameter of RC \\
$p$ & Public system parameter, large prime number \\
$G K$ & Group key shared between RC and registered servers \\
$t_{n}=T_{n}(x)$ & Chebyshev polynomial of degree $n$ \\
$t_{n m}=T_{n}\left(T_{m}(x)\right)$ & Chebyshev polynomial of degree $n$ with input $T_{m}(x)$ \\
$\left(i, t_{i}=T_{i}(x)\right)$ & Secret and public key of the user \\
$\left(r, t_{r}=T_{r}(x)\right)$ & Secret and public key of the RC \\
$\left(s, t_{s}=T_{s}(x)\right)$ & Secret and public key of the server \\
$I D i, I D D_{S}$ & Identity of the user $U$ and server $S$ \\
$T_{i}$ & Timestamp at the registration time of $U$ with CA \\
$c e r t_{i}$ & Certificate on $\left(I D i, t_{i}, T_{i}\right)$ of $U$ by CA \\
$P W, B i o$ & Password and biometrics data of the user $U$ \\
$\epsilon$ & Maximum allowed delay between submission and reception \\
$E_{K}(),. D_{K}()$. & Symmetric key encryption and decryption with key $K$ \\
$H()$. & Hash function \\
$\|, \oplus$ & Concatenation, Bitwise XOR operation \\
\hline
\end{tabular}

all smart phones of the users, servers, RC and CA are time synchronized, as timestamps are used to avoid replay attacks.

\subsection{Notations}

Table 3 summarizes the most important abbreviations and notations used in this paper.

\section{Protocol EAAM}

We distinguish five main phases in the protocol: registration with $\mathrm{CA}$, registration with server, login phase, authentication and session key establishment, and renewal of user's password.

\subsection{Registration with CA}

We need to distinguish actions performed by the RC, the user and its device, and the servers.

\subsubsection{Actions to be performed by the $R C$}

Let $y$ be a secret parameter of the RC. We assume the RC has published the following system parameters: 
$-x$ as the seed for generating the Chebyshev chaotic map

$-p$ as a large prime number, defining the modulo operation on the output of the Chebyshev chaotic map

- $t_{r}=T_{r}(x)$, the public key of the RC, together with a certificate cert $t_{r}$ on $\left(I D_{r}, t_{r}\right)$

- $H(),. E_{K}(),. D_{K}($.$) , representing the hash algorithm, symmetric key en-$ cryption and decryption algorithm with secret shared key $K$

$-\epsilon$ is the predefined delay between submission and reception

\subsubsection{Actions to be performed by the servers}

The server generates its own secret key $s$ and public key $t_{s}$. After approval from the CA, a certificate is generated by the CA. This certificate, together with its identity $I D_{S}$ and public key $t_{s}$ is published in a publicly available repository.

The RC shares with all registered and validated servers a group key $G K$ through a well known multicast group key mechanism, like eg. [52]. This key is regularly updated and in particular when a server is added or removed to the subscription list. Note that the previous versions of the group key, together with the period of their life time, are still stored at the servers until the expiration date.

\subsubsection{Actions to be performed by the user and its device}

Before the user can register, he/she first needs to generate its own key pair $\left(i, t_{i}\right)$ and requests a certificate cert $_{i}$ on the combination of identity $I D_{i}$ and public key $t_{i}$ by the CA. These actions are detailed below.

- Public-private key definition of the user:

The user enters its identity $I D_{i}$, password $P W$ and biometric data Bio into its smart phone. Based on a random variable $r b$ stored in the memory of the phone, the private key $i=H\left(I D_{i}\|P W \oplus r b\| B i o\right)$ is computed. Next, the corresponding public key $t_{i}$ is derived for it. The values $I D_{i}, t_{i}$ are sent to the CA through a secure channel.

- Response of CA:

The CA checks the request by verifying the identity of the user. If positive, it validates the link between $I D_{i}$ and its corresponding claimed public key $t_{i}$ by generating a certificate $\operatorname{cert}_{i}$ on $\left(H\left(I D_{i}, t_{i}, T_{i}\right)\right)$, where $T_{i}$ represents the timestamp at the moment of the registration request.

- Storage on $U_{d}$ :

The device stores the received values $\operatorname{cert}_{i}, t_{i}, T_{i}$, together with the parameter $r b$ and $E_{i}=H\left(P W \oplus r b\|B i o \oplus r b\| I D_{i}\right)$. The parameters $i$ and $I D_{i}$ are stored in the TRM of the device. 


\subsection{Registration with $\mathrm{RC}$}

Using the information $\left(I D_{i}, t_{i}, T_{i}\right.$, cert $\left._{i}\right)$, the user is now able to register with the $\mathrm{RC}$ without the need of a secure channel by performing the following operations. We assume that the public key of the RC can be consulted through secure publicly available repositories. The following steps are executed. The visualization of this process is depicted in Figure 1.

- First, the device computes the value $F_{i}=H\left(i \| I D_{i}\right)$. Then, the user selects a random value $l \in \mathbb{R}$ and computes $t_{l}$. Next, also $t_{l r}$ and $t_{i r}$ are computed, using the public key $t_{r}$ of the RC. Based on the current timestamp $T_{1}$, a dynamic secret $k_{1}$ with the RC can be derived, ie. $k_{1}=H\left(t_{i r}\left\|T_{1}\right\| I D_{i}\right)$. Finally, the key $t_{l r}^{\prime}=H\left(t_{l r} \| T_{1}\right)$ is now used to encrypt the message containing this key $k_{1}$, the value $F_{i}$, and the credentials of the user and the $\mathrm{RC}$, ie. $C_{1}=E_{t_{l r}^{\prime}}\left(k_{1}\left\|F_{i}\right\| I D_{i}\left\|t_{i}\right\| T_{i} \|\right.$ cert $\left._{i} \| t_{r}\right)$. The message $C_{1}, t_{l}, T_{1}$ is sent to the RC.

- Upon arrival, the RC first checks the validity of the timestamp, if the current timestamp $T_{2}$ satisfies $T_{2}-T_{1} \leq \epsilon$. If so, the RC computes $t_{r i}, t_{r l}$ and $t_{r l}^{\prime}$. This last one is used to decrypt $C_{1}$. In order to authenticate the user with identity $I D_{i}$, the certificate cert $_{i}$ is verified and the value of $H\left(t_{i r}\left\|T_{1}\right\| I D_{i}\right)$ is compared with $k_{1}$ from the decrypted message. If all checks are positive, than the RC computes the following parameters. Let $n$ be the number of registrations performed by $I D_{i}$.

$$
\begin{aligned}
A_{i} & =H\left(I D_{i}\|G K\| y \| n\right) \\
B_{i} & =A_{i} \oplus G K \\
C_{i} & =H\left(A_{i}\right) \oplus H\left(F_{i}\left\|t_{i}\right\| I D_{i}\right) \\
k_{2} & =H\left(t_{r l}\left\|T_{1}\right\| T_{2} \| I D_{i}\right) \\
s i g & =\operatorname{sig}_{R C}\left(H\left(T_{2}\|G K\| B_{i} \| t_{i}\right)\right) \\
C_{2} & =E_{k_{2}}\left(B_{i}\left\|C_{i}\right\| T_{2} \| s\right)
\end{aligned}
$$

Here $\left.\operatorname{sig}_{R C}\left(H\left(T_{2}\|G K\| B_{i} \| t_{i}\right)\right)\right)$ denotes the signature of RC over the message $\left.M=H\left(T_{2}\|G K\| B_{i} \| t_{i}\right)\right)$. The message $C_{2}, T_{2}$ is sent to the user.

- After a positive validation of the timestamp, the user computes $k_{2}$ and encrypts $C_{2}$. If $T_{2}$ from this message corresponds with the submitted $T_{2}$, the user stores the parameters $B_{i}, C_{i}, T_{2}$, sig, besides the already stored values $E_{i}, r b$, cert $_{i}, t_{i}, T_{i}$ and $i, I D_{i}$ in TRM. Note that the user is not able to verify the signature, as it does not know the group key $G K$. Instead, the signature verification will be performed later by the servers.

We want to note that each parameter in the derivation of $\mathrm{RC}$ has a specific goal, as will be later shown in more detail in the security analysis. To be short, $A_{i}$ is meant to be only constructible by the RC (knowledge of $y$ ) and $C_{i}$ by the user (knowledge of $F_{i}$ and thus $i, I D_{i}$ ). The user does not know $A_{i}$, but given $B_{i}$ and $H\left(A_{i}\right)$ (from $C_{i}$ ), only the servers knowing $G K$ can verify this link and thus identify a valid user. 


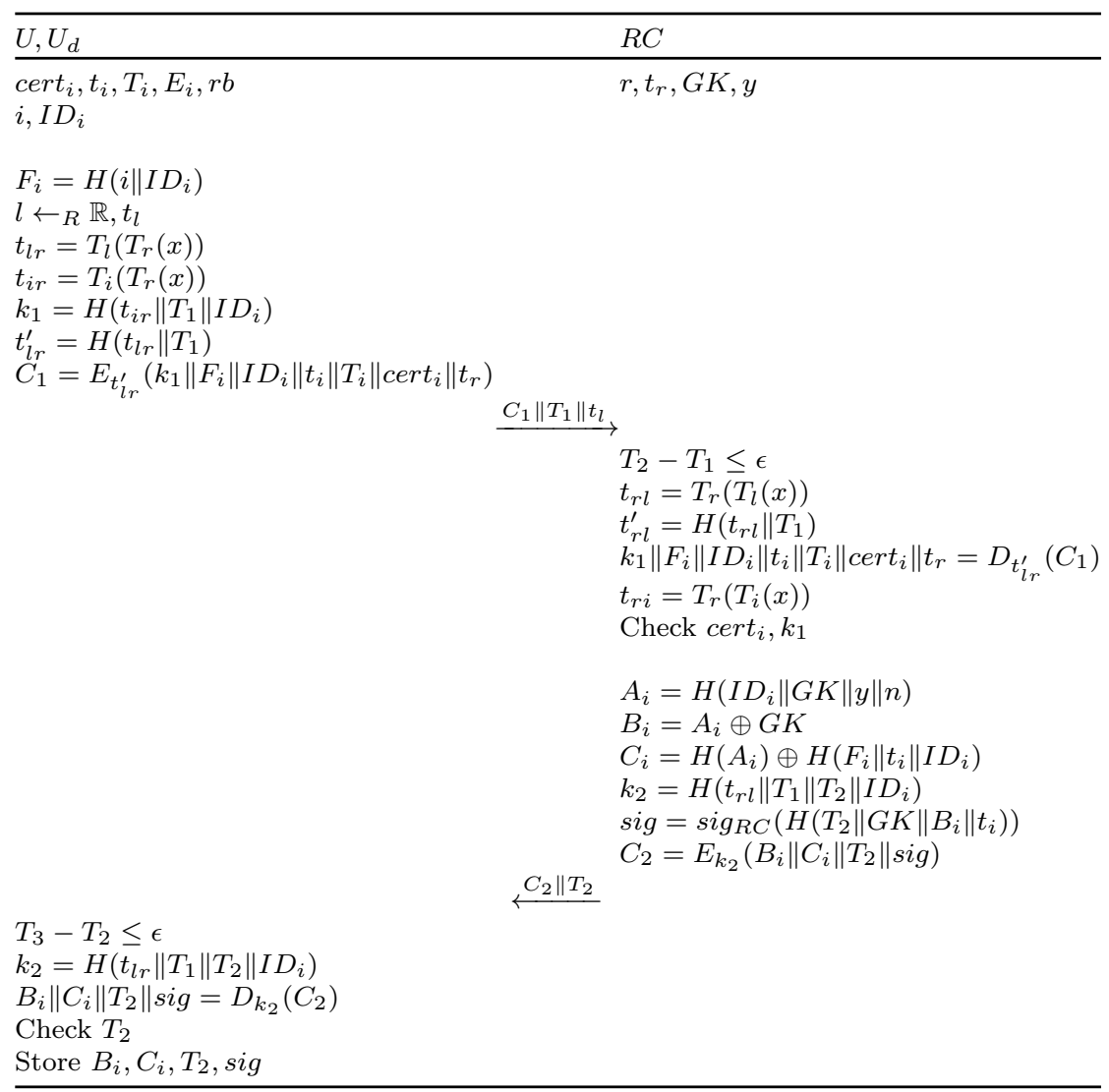

Fig. 1: Steps and computations during the registration request of user with $\mathrm{RC}$

4.3 User login

The user takes its smart phone and inputs its identity $I D_{i}$, password $P W_{i}$, and biometric data Bio. If $H\left(P W \oplus r b\|B i o \oplus r b\| I D_{i}\right)$ corresponds with the stored $E_{i}$, the process can continue. Then, the smart phone computes $F_{i}=H\left(i \| I D_{i}\right)$. It looks up the public key $t_{s}$ of the server $\mathrm{S}$ in a publicly available secured repository. Next, the device picks up a random value $f$ and computes $t_{f}$ and $t_{f s}$. Now the following computations are performed in order to derive the user 
login request to be transmitted, with $T_{3}$ the current timestamp.

$$
\begin{aligned}
H\left(A_{i}\right) & =C_{i} \oplus H\left(F_{i}\left\|t_{i}\right\| I D_{i}\right) \\
C I D_{i} & =B_{i} \oplus H\left(t_{f s} \| T_{3}\right) \\
k_{3} & =H\left(T_{3} \| t_{f s}\right) \oplus H\left(A_{i}\right) \\
C_{3} & =E_{k_{3}}\left(\text { sig }\left\|t_{i}\right\| T_{2}\right)
\end{aligned}
$$

Consequently, the message $t_{f}, T_{3}, C I D_{i}, C_{3}$ will be transmitted to $\mathrm{S}$.

\subsection{Authentication phase and key agreement}

Upon arrival of the message at $T_{4}$ from the user login phase, the server first checks if the timestamp of the user is valid, meaning $T_{4}-T_{3} \leq \epsilon$. If so, $t_{s f}$ will be computed and the following operations are performed.

$$
\begin{aligned}
B_{i} & =C I D_{i} \oplus H\left(t_{s f} \| T_{3}\right) \\
A_{i} & =B_{i} \oplus G K \\
k_{3} & =H\left(T_{3} \| t_{f s}\right) \oplus H\left(A_{i}\right) \\
\operatorname{sig}\left\|t_{i}\right\| T_{2} & =D_{k_{3}}\left(C_{3}\right) \\
M & =H\left(T_{2}\|G K\| B_{i} \| t_{i}\right)
\end{aligned}
$$

If $\operatorname{sig}_{R C}(M)$ corresponds with $\operatorname{sig}$ of $C_{3}$, the server is guaranteed that the credentials are derived by the RC and not by a potential malicious server. However, note that it might still be possible that a malicious server has stored the signatures of previous requests of its users and tries to abuse it in the name of a user. Therefore, it is essential that the SK should also exploit the usage of the public key $t_{i}$. This symmetric shared key equals $S K=H\left(t_{b f}\left\|t_{b i}\right\| t_{s f}\left\|T_{3}\right\| T_{4}\right)$, where $b$ is a randomly chosen parameter and $t_{b}$ the corresponding public variant of it. Finally, $C_{4}=H\left(S K\left\|t_{b f}\right\| t_{b i}\left\|t_{s f}\right\| T_{3} \| T_{4}\right)$ is computed and $C_{4}, t_{b}, T_{4}$ is sent to the user. Note that it is possible to add some restrictions, being that the delay between user login $T_{3}$ and reception of credentials $T_{2}$ is in a predefined range.

With this information, the user's device first verifies the timestamp $T_{4}$. Next, $U_{d}$ computes $t_{i b}, t_{f b}$, and thus also $S K$. Finally, if the hash value of $H\left(S K\left\|t_{f b}\right\| t_{i b}\left\|t_{f s}\right\| T_{3} \| T_{4}\right)$ corresponds with the received $C_{4}$, then user and server have a common shared secret key $S K$. tamper The different steps from the user login and the authentication and key agreement phase are visualized in Figure 2.

\subsection{Password change phase}

If the user wants to change its password, it can complete the procedure without involvement of the RC. The user first logs in with identity and password. The 


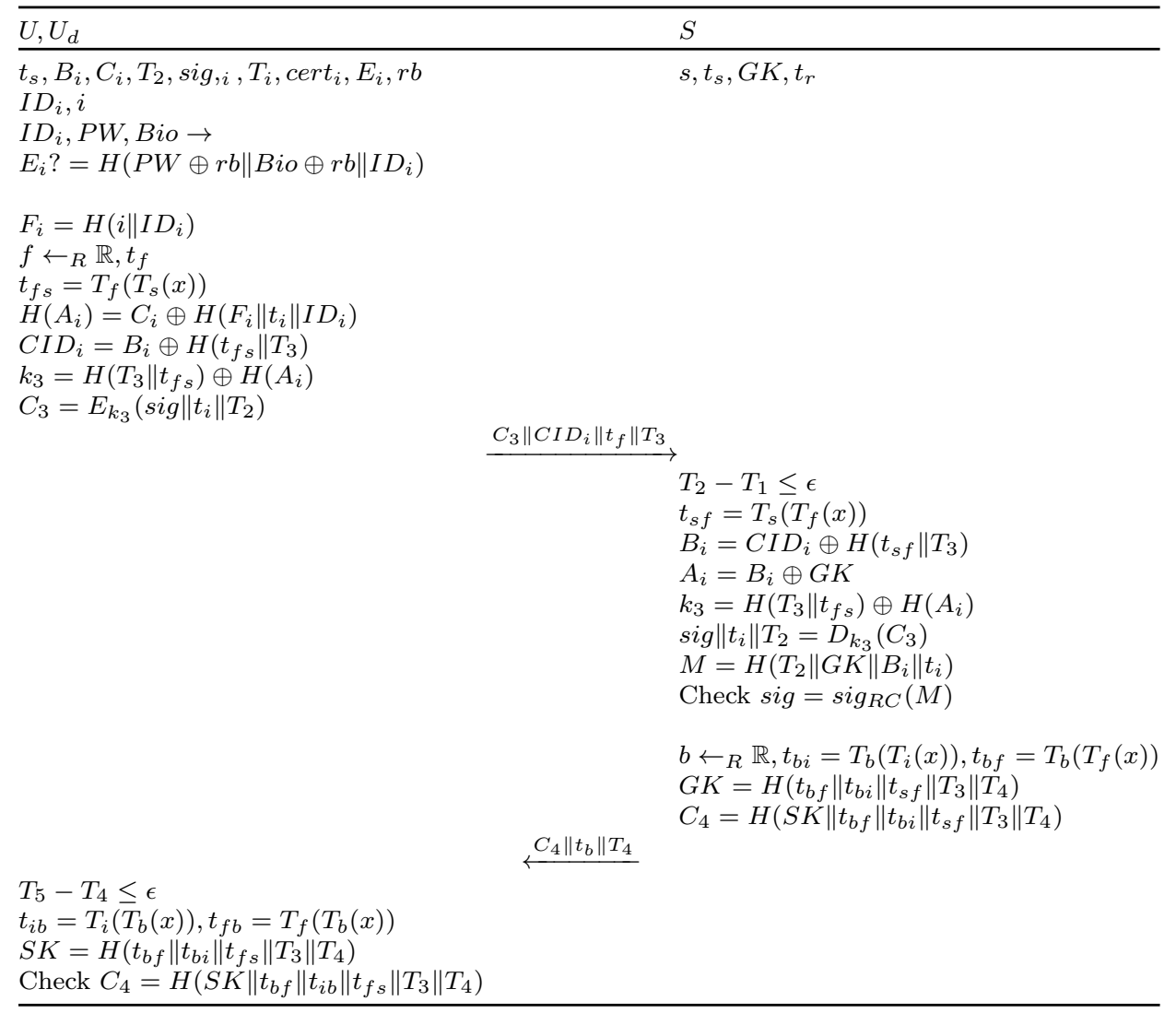

Fig. 2: Steps and computations during the user login and authentication and key agreement phase

device checks the entered information with $E_{i}$. If the user is authentic, the device prompts the user for a new password $P W^{*}$ and computes

$$
\begin{aligned}
r b^{*} & =P W \oplus P W^{*} \oplus r b \\
i^{*} & =i=H\left(I D_{i}\left\|P W^{*} \oplus r b^{*}\right\| B i o\right) \\
t_{i}^{*} & =t_{i}=T_{i^{*}}(x) \\
E_{i}^{*} & =H\left(P W^{*} \oplus r b^{*}\left\|B i o \oplus r b^{*}\right\| I D_{i}\right) \\
F_{i}^{*} & =F_{i}=H\left(i^{*} \| I D_{i}\right) \\
C_{i}^{*} & =C_{i}=H\left(A_{i}\right) \oplus H\left(F_{i}^{*}\left\|t_{i}^{*}\right\| I D_{i}\right)
\end{aligned}
$$

Finally, the values of $E_{i}, r b$ are updated by $E_{i}^{*}, r b^{*}$. The other values remain unchanged.

To conclude, Figure 3 depicts the flow of the different phases, (1) Registration phase with CA, (2) Registration phase of user with RC, (3) User login, 
(4) Authentication and key agreement phase, (5) Password change phase. The key material stored in the different entities is denoted in red.

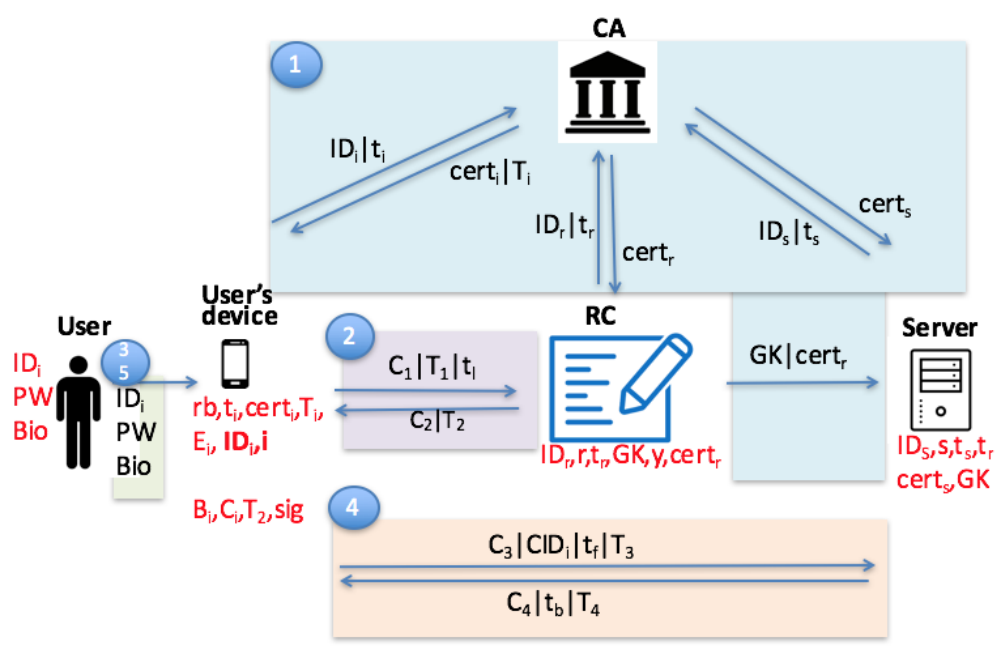

Fig. 3: Steps and computations during the registration request of user with $\mathrm{RC}$

\section{Security analysis}

We focus the security analysis on the user login, authentication and key agreement phase, which is the core of the system.

\subsection{Mutual authentication}

Mutual authentication means that both entities authenticate each other in the protocol. We distinguish the authentication of $\mathrm{S}$ with $\mathrm{U}$ and the other way around.

- S with U: Only the specific $\mathrm{S}$ can derive $B_{i}$ from $C I D_{i}$, by the computation of $t_{f s}$ using its private key $s$. In addition, it can check the validity of the request by verifying the relation with $A_{i}=B_{i} \oplus G K$ and checking the validity of the key $k_{2}$ such that the last part of the decryption of $C_{3}$ corresponds with the public key included in the signature sig. In order to check that the request is really validated by the $\mathrm{RC}$ and not a malicious server knowing the GK, the signature of the RC on the identity (public key $t_{i}$ ) and the timestamp is required to be included in the message and to be verified. As a malicious server can also store the signatures of the 
previous requests of its users, the symmetric shared key between $\mathrm{S}$ and $\mathrm{U}$ also includes $t_{b i}$, which requires the knowledge of the private key $i$ or the randomly defined parameter $b$ by S. Consequently, the server is guaranteed that only a valid user is able to derive the symmetric shared key SK.

- U with S: As U makes use of the public key of S, he is sure that only the entity knowing the corresponding private key is able to derive the required parameters, due to the DL problem. Moreover, the message also implicitly includes $G K$, in both the signature and the definition of $A_{i}$.

\subsection{Impersonation attacks and man-in-the-middle attacks}

This attack implies that an attacker listening to the messages between the intended partners would intercept the communication and would take over the role as legitimate participant. Due to the intensive usage of the public keys of the server and the user on the one hand and the key material derived by the $\mathrm{RC}$ from the other hand, it is impossible for an outsider to derive useful information from the messages or to construct legitimate alternatives.

\subsection{Insider attacks}

Insider attacks are attacks where the involved parties try to abuse the system by sending fake but legitimate requests, allowing the derivation of a secret shared key. In this protocol, we need to distinguish according to the two roles of the user and the server.

A malicious server is able to send a valid user login request, using the stored signature of a previous user request. However, he is not able to derive a symmetric shared secret key as this key involves $t_{b i}$, which requires the knowledge of the private key of the user. Therefore, a malicious server is not able to mimic a user. He also cannot derive any useful information of the messages from the login phase, since both $V_{1}$ and $C I D_{i}$ integrate the parameter $t_{f s}$, only derivable by the server $\mathrm{S}$.

Finally, also a malicious user is not able to generate fake credentials, as he is not aware of the $G K$ or the private key of $\mathrm{RC}$ to make a valid signature.

\subsection{Replay attacks}

A replay attack can be launched when an attacker replays the original message or parts of it at a later moment in time in order to betray or impersonate one of the involved parties. The messages sent in our protocol are $C_{3}\left\|V_{1}\right\| C I D_{i}\left\|t_{f}\right\| T_{3}$ and $C_{4}\left\|t_{b}\right\| T_{4}$. Replay attacks on the first message are impossible due to the usage of the timestamp $T_{3}$, which is included in the other parameters $C_{3}\left\|V_{1}\right\| C I D_{i}$. Similar for the second message, the timestamp $T_{4}$ is included in the computation of $C_{4}$. 
5.5 Known key attacks

These type of attacks allow the derivation of the private keys of $\mathrm{S}$ or $\mathrm{U}$ from the session keys. Since $S K=H\left(t_{b f}\left\|t_{b i}\right\| t_{f s}\left\|T_{3}\right\| T_{4}\right)$ is the result of the hash of $t_{b f}, t_{b i}, t_{f s}$, it is impossible to derive information on $i$ or $s$, due to the one way property of the hash function and in addition due to the $\mathrm{CDH}$ problem.

\subsection{Perfect forward secrecy}

Even if the secret keys of the user or the server are leaked, the previously generated secret session keys remain secret. This follows from the fact that $t_{f b}$ is impossible to derive, without knowledge of $b$ and $f$ and these temporarily generated values are erased from the memories of $U_{d}$ and $\mathrm{S}$ after usage.

5.7 Resistance against password guessing/stolen smart card attacks

In this type of attacks, an attacker might steal the smart phone and use all information available on the phone (eg. by using power analysis techiques $[53,54])$ together with all public messages sent over the public channel. The proposed protocol is secure against such attack as it is a three-factor based protocol, meaning that the knowledge of the password, the possession of the device, and the inheritance of the biometric data should be present with the user for a successful login. Consequently, an attacker in the possession of $U_{d}$, but without knowledge of the password of the biometric data, is not able to activate the phone.

Even if a malicious person gets access to the device and succeeds in deriving the stored parameters $E_{i}, B_{i}, C_{i}, t_{i}, T_{2}, s, r b$, cert $_{i}$. He still does not know the variables $i, I D_{i}$ in the TRM of the phone. These parameters are required to compute $t_{i r}$ and thus $k_{1}$ in the registration request to the RC. Also $i$ and $I D_{i}$ are required in the user login and key agreement with the server to compute $F_{i}$, and thus $H\left(A_{i}\right)$.

\subsection{Prone to curious $\mathrm{RC}$}

A curious and honest $\mathrm{RC}$ is one that follows the required procedures but wants in the end to derive useful information of tis users for its own purpose. The session key in EAAM is only derivable by the involved user and server, as explained above. In particular, the RC is not able to derive the key SK, since SK includes the parameters $t_{b f}, t_{b i}, t_{f s}$, which can due to the $\mathrm{CDH}$ problem only be generated with the knowledge of either the private keys or the randomly derived parameters of the participants. 


\subsection{Anonymity}

Anonymity provides privacy for the users. Anonymity to any outsider is clearly obtained as none of the transmitted messages contain useful information which can link to the identity of the user. Also unlinkability is established due to the usage of a dynamic identity $C I D_{i}$.

Anonymity of the identity of the user by the server is obtained in the case the server is not able to link the public key $t_{i}$ to a specific server. In case the server needs to reveal the identity of the user, the user sends its identity $I D_{i}$ instead of its public key $t_{i}$ in $C_{3}$ during the user login request. In this case, the server needs to get access to a publicly secure directory containing the tuples identity and their corresponding public key.

Note that in none of the cases, the unlinkability of the user by the server can be obtained, without constantly changing the certificate of the user.

\subsection{Access bounded in time}

Due to the fact that the certificate includes a timestamp $T_{2}$, the server has knowledge on the existence date of the certificate and make a decision of the validity on it based on the delay between the request time $T_{3}$ and $T_{2}$.

\subsection{Summary}

We now compare our scheme with the three most efficient systems [42-44] in literature using Chebyshev polynomials, which also only require 2 communication rounds in the authentication request and satisfy most of the required security properties.

None of them satisfy the property of resistance against a curious RC. In addition, [42] is not resistant to insider attacks with malicious servers, and [43] does not satisfy unlinkability. Both $[43,44]$ work with a certificate revocation list (CRL) for bounding the access to privileged non revoked users, while [42] does not consider this aspect.

In EAAM, the access of users is only limited in time (depending on the validity period of the signature of $\mathrm{RC}$ determinable by each individual server). The advantage of this approach, compared with the usage of a CRL on the $\mathrm{RC}$ 's end is that in this case no permanent communication between RC and S is required and a differentiated approach to each server is possible. Moreover, in most practical scenarios, the certificate is valid upon a certain amount of time.

Finally, the last difference is that [42] is a 2 factor based authentication protocols, while EAAM and $[43,44]$ are all 3 factor based, including biometric information.

To conclude, Table 2 summarizes the comparison of the most challenging security features and shows the difference between our protocol EAAM and $[42-44]$. 
Table 2: Comparison of security features

\begin{tabular}{lllllll}
\hline Protocol & $\begin{array}{l}\text { Anony- } \\
\text { mity }\end{array}$ & $\begin{array}{l}\text { No insider } \\
\text { attacks }\end{array}$ & $\begin{array}{l}\text { Perfect forward } \\
\text { secrecy }\end{array}$ & $\begin{array}{l}\text { Unlink- } \\
\text { ability }\end{array}$ & $\begin{array}{l}\text { Curious } \\
\text { RC }\end{array}$ & $\begin{array}{l}\text { No secure } \\
\text { channel(RC-U) }\end{array}$ \\
\hline EAAM & + & + & + & + & + & + \\
{$[42]$} & + & - & + & + & - & - \\
{$[43]$} & + & + & - & - & - & - \\
{$[44]$} & + & + & + & + & - & - \\
\hline
\end{tabular}

Table 3: Comparison of the communication requirements

\begin{tabular}{lll}
\hline Protocol & Number of transmitted bits & Number of received bits \\
\hline EAAM & 608 & 224 \\
{$[42]$} & 384 & 224 \\
{$[43]$} & 640 & 640 \\
{$[44]$} & 384 & 224 \\
\hline
\end{tabular}

\section{Performance analysis}

We focus on the operations and communication requirements from the side of the user, as it is the most critical in the computational point of view. Moreover, we limit the discussion to the user login and authentication request phase as the registration phase is only performed once. Moreover, our system does not require a secure channel between the user and the $\mathrm{RC}$, while the others need such channel to share secret parameters.

\subsection{Communication requirements}

We assume, similar to [42-44], that the number of bits for the identity and timestamps equals to 32 . The number of bits as a result of the hash operation is 160 bits (eg. SHA 1) and the result of encryption and decryption is a multiple of 128 bits (eg. AES). The comparison of the number of transmitted and received bits are summarized in the Table 3 . We can conclude that our protocol requires an additional 224 bits to send a message, compared with the best protocols $[42,44]$. This follows from the fact that we need to include the signature of the CA. Note that we have a very compressed signature as the message to be signed is already derived from the previous parameters. Only two auxiliary variables for the signature generation need to be included in sig. The number of received bits is equal to the best performing systems in literature.

\subsection{Computational requirements}

The operations to be considered in the comparison are the hash operation, bio hash operation, symmetric encryption and decryption algorithm, and the 
Table 4: Computation time of the operations [57]

\begin{tabular}{lll}
\hline Operation & Description & Time (in ms) \\
\hline$T_{H}$ & Time for SHA 1 Hash operation & 0.1 \\
$T_{B H}$ & Time for bio hash operation & 1.7 \\
$T_{E}$ & Time for encryption or decyrption with AES 128 & 0.1 \\
$T_{C}$ & Time for Chebyshev polynomial map evaluation & 0.12 \\
\hline
\end{tabular}

Table 5: Comparison of the performance

\begin{tabular}{lll}
\hline Protocol & Computation cost & Total time (in ms) \\
\hline EAAM & $7 T_{H}+1 T_{B H}+1 T_{E}+4 T_{C}$ & 3.12 \\
{$[42]$} & $5 T_{H}+3 T_{C}$ & 0.96 \\
{$[43]$} & $11 T_{H}+1 T_{B H}+2 T_{E}+4 T_{C}$ & 3.7 \\
{$[44]$} & $6 T_{H}+1 T_{B H}+4 T_{C}$ & 2.9 \\
\hline
\end{tabular}

Chebyshev polynomial evaluation. The xor operation is negligible, compared with these operations. In [55], the authors state that the time for executing a biohashing operation is similar to the time required for elliptic curve multiplication. Also the time to evaluate a Chebyshev polynomial can be approximated by the time corresponding with the execution of a hashing operation [56]. Table 4 shows the execution time for each of these operations as derived in [57]. The measurements are performed on a $2260 \mathrm{MHz}$ ARM device, corresponding with a smartphone with Android OS (Android 4.4 KitKat), containing a 32-bit processor, ARMv7 Quadcore. The timing values are measured using the system clock. The test applications are written in the JAVA programming language by using the Android Software Development Kit (SDK) and the available crypto APIs. The resulting performance value is obtained after taking the average of 10 iterations.

As a result, Table 5 compares the performance of EAAM with the systems [42-44], both in terms of computation cost and in total time. Note that all these schemes consist of two communication rounds in the authentication request of the user to the server and are based on Chebyshev polynomials.

We can conclude that the most efficient protocol [42] is almost double efficient as the others. This follows from the first place since it is a two-factor based protocol, and thus not requires a bio hash operation (corresponding to the most compute intensive operation). Next, the protocol of [42] also satisfies a clear weakness in a sense that it is not secure against insider attacks of malicious servers and can in fact from a security point of view be seen as only a single server system. The main difference between our protocol and [44] is an additional encryption and hash for the derivation of the encryption key, which is required to send the certificate of the CA. As the protocols require less than $350 \mathrm{~ms}$ in general, they can be considered as real time from a user's point of view [57]. 


\section{Conclusion}

This paper presents an efficient anonymous authentication protocol in multiple server communication networks, called EAAM. In the EAAM, the user required three-factors (e.g., knowledge of password, possession of biometrics and smartphone) for accessing multiple servers. The main novelty of the proto$\mathrm{col}$ is that it provides resistance against an honest but curious RC. Especially with respect to the privacy concerns of the user, it is very important to know that no big brother in the system exists which is able to collect or analyse all information of the different users to be exploited for different purposes without awareness of the user. Moreover, EAAM also offers very elegantly a differentiated validity period of its access permission delivered by the RC for the different servers and does not work by means of a CRL requiring additional communication between $\mathrm{RC}$ and $\mathrm{S}$ during the authentication request.

We have discussed the security strengths of the system and have shown that the protocol is very competitive with respect to computational and communication requirements, compared with the state of the art and taken into account its additional security features.

\section{References}

1. Hwang, M., Li, L., A new remote user authentications scheme using smart cards, IEEE Transactions on Consumer Electronics, 46(1), 28-30 (2000). Li, L., Peng, H., Kurths, J., Yang, Y., Schellnhuber, H.J.:

2. He, D., Zeadally, S., Wang, H., Liu, Q., Lightweight Data Aggregation Scheme against Internal Attackers in Smart Grid Using Elliptic Curve Cryptography, Wireless Communications and Mobile Computing, 2017, 11 pages, (2017).

3. Li, L., Peng, H., Kurths, J., Yang, Y., Schellnhuber, H.J., Chaos-order transition in foraging behavior of ants, PNAS 111(23), 8392-8397 (2014).

4. Tashi, J., J., Comparative analysis of smart card authentication schemes, IOSR Journal of Computer Engineering, 16(1), 91-97 (2014).

5. Huang, X., Xiang, Y., Chonka, A., Zhou, J., Deng, R.H., A generic framework for threefactor authentication: preserving security and privacy in distributed systems, IEEE Trans. Parallel Distrib. Syst. 22(8), 1390-1397 (2011).

6. Dodis Y., Reyzin L., Smith A., Fuzzy extractors: How to generate strong keys from biometrics and other noisy data, Proceedings of EUROCRYPT, 523-540 (2004).

7. Hsieh, W. B., Leu, J. S., An anonymous mobile user authentication protocol using selfcertified public keys based on multi-server architectures, Journal of Supercomputing, 70(1) 133-148 (2014).

8. Zhao, D., Peng, H., Li,Y. Y. S., An efficient dynamic ID based remote user authentication scheme using self-certified public keys for multi-server environment, CoRR abs/1305.6350, (2013).

9. Tseng, Y. M. , Wu, T. Y., Wu, J., A pairing-based user authentication scheme for wireless clients with smart card. Informatics, 19(2), 285 ?302 (2008).

10. Geng, J., Zhang, L., A dynamic id-based user authentication and key agreement scheme for multi-server environment using bilinear pairings, Workshop on Power Electronics and Intelligent Transportation System (PEITS 2008), Guangzhou, China, 33 ?37 (2008).

11. Hsu, C. L., Lin, T. W., Password authenticated key exchange protocol for multi-server mobile networks based on chebyshev chaotic map, IEEE International Conference on Pervasive Computing and Communications (PERCOM 2013), San Diego, California, USA, 90?95 (2013). 
12. Jiang, Q., Wei, F., Fu, S., Ma, J., Li, G., Alelaiwi, A., Robust extended chaotic mapsbased three-factor authentication scheme preserving biometric template privacy, Nonlinear Dynamics, 83(4), 2085-2101 (2015).

13. Liao, Y. P., Hsiao, C.M., A novel multi-server remote user authentication scheme using selfcertified public keys for mobile clients, Future Generation Computer Systems, 29(3), 886-900, (2013).

14. Amin, R., Biswas, G.P., Design and Analysis of Bilinear Pairing Based Mutual Authentication and Key Agreement Protocol Usable in Multi-server Environment, Wireless Pers Commun, 84(1), 439-462, (2016).

15. Banerjee, S., Dutta, M.P., Bhunia C.T., An Improved smart card based anonymous multi-server remote user authentication scheme, International Journal of Smart Home, 9(5), 11-22 (2015).

16. Baruah, K.CH., Banerjee, S., Dutta, M.P., Bhunia C.T., An Improved Biometric-based Multi-server Authentication Scheme using Smart Card, International Journal of Security and Its Application, 9(1), 397-408 (2015).

17. Li, C.T., and Hwang, M.S., An efficient biometrics based remote user authentication scheme using smart cards, Journal of Network and Computer Applications, 33(1), 1-5. (2010).

18. Chuang, M.C., Chen, M.C., An anonymous multi-server authenticated key agreement scheme based on trust computing using smart cards and biometrics, Expert Systems with Applications, 41(4), 1411-1418 (2014).

19. Mishra, D., Das, A.K., and Mukhopadhyay, S., A secure user anonoymity-preserving biometric-based multi-server authenticated key agreement scheme using smart cards, Expert Systems with Applications, 41(18), 8129-8143 (2014).

20. Das, A.K., Analysis and improvement on an efficient biometric based remote user authentication scheme using smart cards, IET Information Security, 5(3), 145-151 (2011).

21. An, Y., Security analysis and enhancements of an effective biometric-based remote user authentication scheme using smart cards, Journal of Biomedicine and Biotechnology, 6 pages (2012).

22. Khan, M.K. and Kumari, S, An improved biometrics-based remote authentication scheme with user anonymity, Journal of Biomedicine and Biotechnology, 9 pages (2013).

23. Wen, F., Susilo, W., Yang, G., Analysis and improvement on a biometric-based user authentication scheme using smart cards, Wireless Personal Communications, 80, 17471760 (2015).

24. Braeken, A., Porambage, P., Efficient Anonym Smart Card Based Authentication Scheme for Multi-Server Architecture, 9(9), 177-184 (2015).

25. Pippal, R.S., Wu, S., Robust smart card authentication scheme for multi-server architecture, Wireless Personal Communications, 72(1), 729-745 (2013).

26. Wei, J., Liu, W., Hu, X. Cryptanalysis and improvement of a robust smart card authentication scheme for multi-server architecture, Wireless Personal Communications, 77(1), 2255-2269 (2014).

27. Lin, H. Wen, F., Du, C., A novel and anonymous key agreement multi-server architecture, Journal of Computational Information Systems, 11(8), 3011-3018 (2015).

28. Yoon E., Yoo, K., Robust biometrics-based multi-server authentication with key agreement scheme for smart cards on elliptic curve cryptosystem, The Journal of Supercomputing, 63(1), 235-255 (2013).

29. Kim, H., Jeon, W., Lee, K., Lee, Y., Won, D., Cryptanalysis and improvement of a biometrics-based multi-server authentication with key agreement scheme, Proceedings of 12th International Conference on Computational Science and Its Applications (ICCSA 2012), Salvador de Bahia, Brazil, 391 ? 406 (2012).

30. He, D., Wang, D., Robust Biometrics-Based Authentication Scheme for Multiserver Environment, IEEE Systems Journal, 9(3), 816-823 (2015).

31. Odelu, V., Das, A. K., Goswami, A., A secure biometrics-based multiserver authentication protocol using smart cards, IEEE Transactions on Information Forensics and Security, 10(9), 1953-1966 (2015).

32. Jiang, Q., Khan, M. K., Lu, X., Ma, J., He, D., A privacy preserving three-factor authentication protocol for e-health clouds, The Journal of Supercomputing, 1-24 (2016). 
33. Jiang, Q., Ma, J., Li, G., Yang, L., An Efficient Ticket Based Authentication Protocol with Unlinkability for Wireless Access Networks, Wireless Personal Communications, $77(2), 1489-1506$ (2014).

34. Guo, C., Chang, C.-C., Chaotic maps-based password authenticated key agreement using smart cards, Commun. Nonlinear Sci. Numer. Simul., 18(6), 1433-1440 (2013).

35. Hao, X., Wang, J., Yang, Q., Yan, X., Li, P.: A chaotic map based authentication scheme for telecare medicine information systems. J. Med. Syst., 37(2), 1-7 (2013).

36. Jiang, Q., Ma, J., Lu, X., Tian, Y.: Robust chaotic map based authentication and key agreement scheme with strong anonymity for telecare medicine information systems., J. Med. Syst. 38(2), 1 ?8 (2014).

37. Lee, C.C., Chen, C.C.,Wu, C.Y., Huang, S.-Y., An extended chaotic maps-based key agreement protocol with user anonymity, Nonlinear Dyn. 69(1-2), 79-87 (2012).

38. Lee, C.C., Hsu, C.W., A secure biometric-based remote user authentication with key agreement scheme using extended chaotic maps, Nonlinear Dyn. 71(1-2), 201-211 (2013).

39. Islam, S.K.H., Provably secure dynamic identity-based three-factor password authentication scheme using extended chaotic maps. Nonlinear Dyn. 78(3), 2261-2276 (2014).

40. Khan, M.K., Zhang, J.,Wang, X., Chaotic hash-based fingerprint biometric remote user authentication scheme on mobile devices, Chaos, Solitons Fract. 35(3), 519-524 (2008).

41. He, D., Chen, Y., Chen, J., Cryptanalysis and improvement of an extended chaotic maps-based key agreement protocol, Nonlinear Dyn. 69(3), 1149-1157 (2012).

42. Lee, C.C., Lou, D.C., Li, C.T.: An extended chaotic maps based protocol with key agreement for multiserver environments, Nonlinear Dyn, 76(1), 853-866 (2013).

43. Chatterjee, S. Roy, S., Das, A.K., Chattopadhyay, S., Kumar, N., Vasilakos, A.V., Secure Biometric-Based Authentication Scheme using Chebyshev Chaotic Map for Multi-Server Environment, IEEE Transactions on dependable and secure computing, (2016).

44. Irshad, A., Sher, M., Chaudhary, S.A., Naqvi, H., Farash, M.S., An efficient and anonymous multi-server authenticated key agreement based on chaotic map without engaging Registration Centre, J Supercomput, 72(4), 1623-1644 (2016).

45. Bergamo, P., Arco, P., Santis, A., Kocarev, L., Security of public key cryptosystems based on Chebyshev polynomials, IEEE. Trans. Circ. Syst. 52, 1382-1393 (2005).

46. Kocarev L, Lian S., Chaos-based cryptography: theory, algorithms and applications, ISBN 978-3-642-20542-2, Springer, Berlin, (2011).

47. Zhang, L., Cryptanalysis of the public key encryption based on multiple chaotic systems, Chaos Solitons Fract. 37(3), 669-674 (2008).

48. Chain, K., Kuo, W.C., A new digital signature scheme based on chaotic maps, Nonlinear Dynamics, 74 (4), 1003-1012 (2013).

49. Dodis, Y., Reyzin, L., and Smith, A., Fuzzy extractors: How to generate strong keys from biometrics and other noisy data, Eurocrypt (2004).

50. Jin, A., Ling, D., and Goh, A., Biohashing: two factor authentication featuring fingerprint data and tokenised random number, Pattern Recognition, 37(11), 2245-2255 (2004).

51. Ratha, N. K., Connell, J. H., Bolle, R. M., Enhancing security and privacy in biometricsbased authentication systems, IBM Systems Journal, 40(3), 614 - 634 (2001).

52. Porambage, P., Braeken, A. , Schmitt, C., Gurtov, A.V., Ylianttila, M., Stiller, B., Group Key Establishment for Enabling Secure Multicast Communication in Wireless Sensor Networks Deployed for IoT Applications, IEEE Access 3, 1503-1511 (2015).

53. Messerges, T. S., Dabbish, E. A., Sloan, R. H., Examining smart-card security under the threat of power analysis attacks, IEEE Transactions on Computers, 51(5) 541-552 (2002).

54. Kocher, P., Jaffe, J., Jun, B., Differential power analysis, Proceedings of Advances in Cryptology - CRYPTO99, LNCS, vol. 1666. Santa Barbara, California, USA, Springer, 388-397 (1999).

55. He, D., Kumar, N., Lee, J. H., Sherratt, R. S., Enhanced three- factor security protocol for consumer USB mass storage devices, IEEE Trans. Consum. Electron., 60(1): 30 ?37 (2014).

56. Lee, T. F.,Provably secure anonymous single-sign-on authentication mechanisms using extended Chebyshev chaotic maps for distributed computer networks. IEEE Syst. J., (2015).

57. Malina, Hajny, J., Fujdiak, R., Hosek, J., On perspective of security and privacypreserving solutions in the internet of things, Computer Networks, 102 (19), 83-95, (2016). 